\title{
Data report: clast counts and petrography of gravels from Site C0007, IODP Expedition 316, Nankai Trough'
}

\author{
Christopher L. Fergusson²
}

\section{Chapter contents}

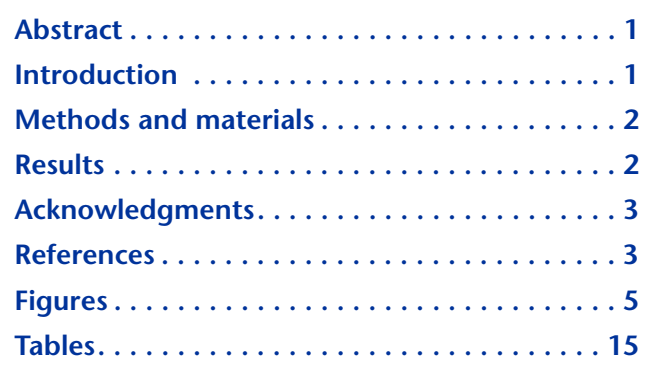

${ }^{1}$ Fergusson, C.L., 2011. Data report: clast counts and petrography of gravels from Site C0007, IODP Expedition 316, Nankai Trough. In Kinoshita, M., Tobin, H., Ashi, J., Kimura, G., Lallemant, S., Screaton, E.J., Curewitz, D., Masago, H., Moe, K.T., and the Expedition 314/315/316 Scientists, Proc. IODP, 314/315/316: Washington, DC (Integrated Ocean Drilling Program Management International, Inc.).

doi:10.2204/iodp.proc.314315316.203.2011

${ }^{2}$ School of Earth and Environmental Sciences, University of Wollongong, Wollongong NSW 2522, Australia.cferguss@uow.edu.au

\section{Abstract}

Gravel beds drilled during Integrated Ocean Drilling Program Expedition 316 at Site C0007 in the eastern Nankai Trough, 100 $\mathrm{km}$ offshore from the Kii Peninsula of Honshu, southwest Japan, have been investigated using 26 samples from two horizons (Cores 316-C0007C-17H and 316-C0007D-12R). The gravel from Core $316-\mathrm{C} 0007 \mathrm{C}-17 \mathrm{H}$ is from a $1.7 \mathrm{~m}$ thick layer, whereas the gravel from Core 316-C0007D-12R is from a layer $4.55 \mathrm{~m}$ thick. The upper parts of both layers are normally graded. The gravel is clast-supported, polymictic, of granule to pebble size, moderately to poorly sorted, and with subrounded to angular fragments. Clast types are similar from both cores with abundant finegrained sedimentary clasts that are well cemented, and many clasts contain small quartz veins indicative of a source with a very low metamorphic grade. The main sedimentary clast types are chert of various colors (black, gray, light yellow-brown, clear, and rare red and purple), siltstone, and sandstone. Other clast types are vein quartz and igneous clasts of mainly intermediate volcanic composition, minor plutonic rock fragments, and minor metamorphic rock fragments. Intraformational soft mud fragments occur in most samples.

\section{Introduction}

Expedition 316 of the Integrated Ocean Drilling Program (IODP) undertook drilling and sampling at Site C0007 $\left(33^{\circ} 01.23258^{\prime} \mathrm{N}\right.$, $136^{\circ} 47.94852^{\prime} \mathrm{E}$ ) along the frontal thrust in the toe region of the Nankai Trough accretionary prism as part of the Nankai Trough Seismogenic Zone Experiment (NanTroSEIZE) offshore from the Kii Peninsula, Honshu, southwest Japan. The NanTroSEIZE project aims to establish controls on earthquake and tsunami genesis by drilling and sampling sediments and rocks in the eastern Nankai Trough. At Site C0007, turbidites and hemipelagic mud were sampled and the stratigraphy consisted of an overall coarsening and thickening upward succession with deep-marine basin deposits of the Shikoku Basin at the base overlain by trench wedge deposits, which are in turn overlain by lower slope deposits (see Expedition 316 Scientists, 2009a). Four gravel layers were encountered in channelized deposits of the upper trench wedge: Cores 316-C0007C-17H (157.09-159.125 m core depth below seafloor 
[CSF]) and 316-C0007D-3R (190-190.6 m CSF), 316C0007D-11R (266.5-266.6 m CSF), and 316-C0007D12R (275.6-280.1 m CSF) (Fig. F1) (see Expedition 316 Scientists, 2009a). Samples from the two thicker gravel-bearing intervals (9 samples from Core 316C0007C-17H and 17 samples from Core 316-C0007D12R; Fig. F2) have been counted for clast composition with accompanying petrographic study and geochemical analyses.

\section{Methods and materials}

Samples were requested postcruise and were initially selected evenly spaced throughout the gravel intervals. Some samples were taken at different places than those requested because of voids, sample depletion, and the local poor condition of the core. Bulk 4 $\mathrm{cm}$ long intervals were requested. Samples 316C0007D-12R-5, 15.5-19.5 cm; 12R-5, 39.5-43.5 cm; and $12 \mathrm{R}-5,55-59 \mathrm{~cm}$, had volumes of $120 \mathrm{~cm}^{3}$; Samples $12 \mathrm{R}-\mathrm{CC}, 7-11 \mathrm{~cm}$, and $12 \mathrm{R}-\mathrm{CC}, 51-55 \mathrm{~cm}$, had volumes of $50 \mathrm{~cm}^{3}$ and all other samples had volumes of $20 \mathrm{~cm}^{3}$. Some of the samples from Core 12R contained some mud matrix that was removed by washing the sample on a $2 \mathrm{~mm}$ mesh sieve. It is possible that some intraformational soft mud fragments were washed away during sieving and others may have disintegrated into smaller pieces. Intraformational mud fragments were counted although it is possible that some fragments may have been unintentionally broken into smaller pieces during handling prior to counting. It should also be noted that significant disturbance of the gravels during drilling and retrieval of core may have occurred.

A subsample was poured from each of the 19 sample bags from Core 316-C0007D-12R into a $2.5 \mathrm{~cm}$ diameter plastic cylindrical cup. Blue-stained epoxy resin was poured into the container and allowed to harden. One thin section was made for each of these samples. Only two thin sections were made from Core 316C0007C-17H; one each from Samples 17H-1, 34-38 $\mathrm{cm}$, and $17 \mathrm{H}-2,49-53 \mathrm{~cm}$. These two thin sections were made from a grain mount consisting of selected different clast types rather than an unsorted subsample, as was the case for all the thin sections from Core 316-C0007C-12R. Thin sections were made at the University of Wollongong (Australia) by standard thin section techniques.

After an extensive initial examination of the samples, a common set of clast categories was determined for all samples from Core 316-C0007C-17H and a slightly different set of categories was used for all samples from Core 316-C0007D-12R. This is be- cause the gravels from Core 316-C0007D-12R are finer grained and it is more difficult to distinguish some clast types, such as chert, from very finegrained sedimentary rock fragments. For each sample, clasts were divided into categories and clasts were counted. Identification of clasts types was assisted by using a binocular microscope and/or hand lens. All samples are poorly sorted and samples from Core 316-C0007D-12R include many sand-sized grains that were not removed by sieving through a 2 $\mathrm{mm}$ mesh sieve because of their angularity. Grains on the boundary between very coarse sand and granule grades (i.e., 2.0-2.5 mm) were not counted. Counts of clast types were undertaken on thin sections for samples from Core 316-C0007D-12R. Use of a transmitting light microscope enabled more categories of clast types to be used.

Grain size measurements of maximum grain diameters of 64-204 clasts were determined from all nine samples from Core 316-C0007C-17H.

Six larger clasts, representative of different clast types, from Sample 316-C0007C-17H-CC, 5-9 cm, were analyzed for major elements by X-ray fluorescence (XRF) at the School of Earth and Environmental Sciences, University of Wollongong (analyst Paul Carr).

\section{Results \\ Description of gravel samples}

Site $\mathrm{C0007}$ gravels are clast-supported, granule to fine pebble grade, poorly to moderately sorted, polymictic, and have subrounded to angular clasts. Samples from Core 316-C0007C-17H lack mud matrix, and mud matrix is only locally developed in Core 316-C0007D-12R. In Core 316-C0007C-17H, the gravel is part of a $1.7 \mathrm{~m}$ thick layer that is normally graded in the topmost $1.5 \mathrm{~m}$. In Core 316C0007D-12R, the gravel is from a $4.55 \mathrm{~m}$ thick layer that is normally graded in the topmost $1.5 \mathrm{~m}$. Gravel samples from Core 316-C0007C-17H were notably coarser than samples from Core 316-C0007D-12R. Gravels are of granule to fine pebble size in Core 316-C0007C-17H (Tables T1, T2). In Core 316C0007D-12R the gravel is granule grade with abundant sand-sized grains.

Clast types are similar in both cores. The most common clast types are fine-grained sedimentary rock fragments (Tables T3, T4, T5; Figs. F3, F4, F5). Many of these clasts contain quartz veins. In Core 316C0007D-12R, many clasts are categorized as combined vein quartz and fine-grained sedimentary rock fragments because quartz veins are so abundant 
( $>50 \%$ of the clast). Chert is common and mostly dark gray to black with minor quantities of light-colored yellow-brown and clear chert. In Core 316C0007D-12R, rare clasts are red to purple chert (jasper). Sandstone clasts are also common in samples from both cores. Vein quartz clasts (5\%-26\%) are present in all samples. Igneous clasts are mainly intermediate volcanic rock fragments, with additional minor silicic rock fragments (including pumice), scarce basaltic rock, and minor plutonic rock fragments. Metamorphic rock fragments are also scarce. Light brown intraformational soft mud fragments occur in most samples.

\section{Petrography of clast types}

It is clear from thin sections that all clast types display considerable variation in characteristics. Counts of clast types in the grain mounts from granule-sized gravel are given in Table T5.

Chert ranges from impure varieties to chert consisting almost wholly of silica (Fig. F4A-F4F). Many chert clasts have quartz veins. Radiolarians occur in some fragments (Fig. F4C-F4F). Some chert clasts are strongly recrystallized.

Siltstone is highly variable and includes silty claystone and impure chert. Most fragments are dark colored with fine to abundant veins of quartz and, less commonly, calcite and chlorite. Several of these clast types were chemically analyzed by Expedition 316 Scientists (see Table T7 in Expedition 316 Scientists, $2009 \mathrm{~b}$ ) and were shown to consist mainly of quartz with lesser plagioclase and clay.

Sandstone is also highly varied (Fig. F5). Grain size ranges from very fine to fine sandstone with scarce medium-grained sandstone. Most sandstone clasts consist of quartz, minor plagioclase, and lithic grains (sedimentary, volcanic rock fragments, vein quartz, and plutonic rock fragments). Some lithic sandstone clasts contain radiolarian chert fragments. Pure quartz sandstone and sandstone with calcite cement are rare. Some sandstone clasts contain quartz veins. Rare lithic sandstone clasts show evidence of lowgrade metamorphism.

As noted above for vein quartz, all gradations occur between pure vein quartz and vein quartz containing mostly slivers of sedimentary wall rocks.

Volcanic rock fragments are mainly intermediate in composition with less common silicic and mafic clasts. Intermediate clasts texturally are porphyritic with altered glassy groundmass and abundant phenocrysts of plagioclase and fewer pyroxene pheno- crysts (Fig. F6A-F6D). Silicic volcanic rock fragments are porphyritic with quartz and feldspar phenocrysts in a recrystallized, very fine grained groundmass. Mafic clasts are also porphyritic with pyroxene and plagioclase phenocrysts in an altered glassy groundmass. Some volcanic rock fragments are partially altered to epidote and chlorite.

Plutonic rock fragments are mainly coarse to medium-grained plagioclase-rich clasts of intermediate composition and granitic clasts with quartz and feldspar crystals (Fig. F6E-F6H). Medium-grained to fine-grained holocrystalline clasts of mainly intermediate composition also occur, probably derived from shallow and/or small intrusions.

Metamorphic rock fragments include fine-grained metasedimentary rock fragments containing common fine-grained biotite (Fig. F7) probably derived from contact aureoles adjacent to intrusions.

\section{Chemical analyses}

The chert and vein quartz clasts, as expected, have very high silica values (>94\%). The sandstone and siltstone fragments are also relatively high in silica (77\% and $83 \%$, respectively). A volcanic rock fragment, thought to be of intermediate composition, was higher in silica (72\%) than expected and probably reflects alteration of the sample. The metamorphic rock was thought to be a metamorphosed mafic volcanic rock but it also is significantly higher in silica (71\%) than expected. See Table T6 for X-ray fluorescence $(\mathrm{XRF})$ results.

\section{Acknowledgments}

This study used samples provided by the Integrated Ocean Drilling Program (IODP). Thin sections were made by José Abrantes at the School of Earth and Environmental Sciences, University of Wollongong. Associate Professor Paul Carr kindly arranged the XRF analyses at the University of Wollongong. Dr. Sol Buckman assisted with photographs of clasts in thin sections. The author is grateful for the useful review of Professor Kathleen Marsaglia and editorial input from Lorri Peters (IODP) and Professor Liz Screaton (Co-Chief Scientist, Expedition 316).

\section{References}

Expedition 316 Scientists, 2009a. Expedition 316 Site C0007. In Kinoshita, M., Tobin, H., Ashi, J., Kimura, G., Lallemant, S., Screaton, E.J., Curewitz, D., Masago, H., Moe, K.T., and the Expedition 314/315/316 Scientists, 
Proc. IODP, 314/315/316: Washington, DC (Integrated Ocean Drilling Program Management International, Inc.). doi:10.2204/iodp.proc.314315316.135.2009

Expedition 316 Scientists, 2009b. Expedition 316 methods. In Kinoshita, M., Tobin, H., Ashi, J., Kimura, G., Lallemant, S., Screaton, E.J., Curewitz, D., Masago, H., Moe, K.T., and the Expedition 314/315/316 Scientists, Proc. IODP, 314/315/316: Washington, DC (Integrated Ocean
Drilling Program Management International, Inc.). doi:10.2204/iodp.proc.314315316.132.2009

Initial receipt: 18 July 2010

Acceptance: 28 February 2011

Publication: 11 July 2011

MS 314315316-203 
Figure F1. Stratigraphic columns for Site C0007. Core recovery for Holes C0007A-C0007C. (Continued on next page.)

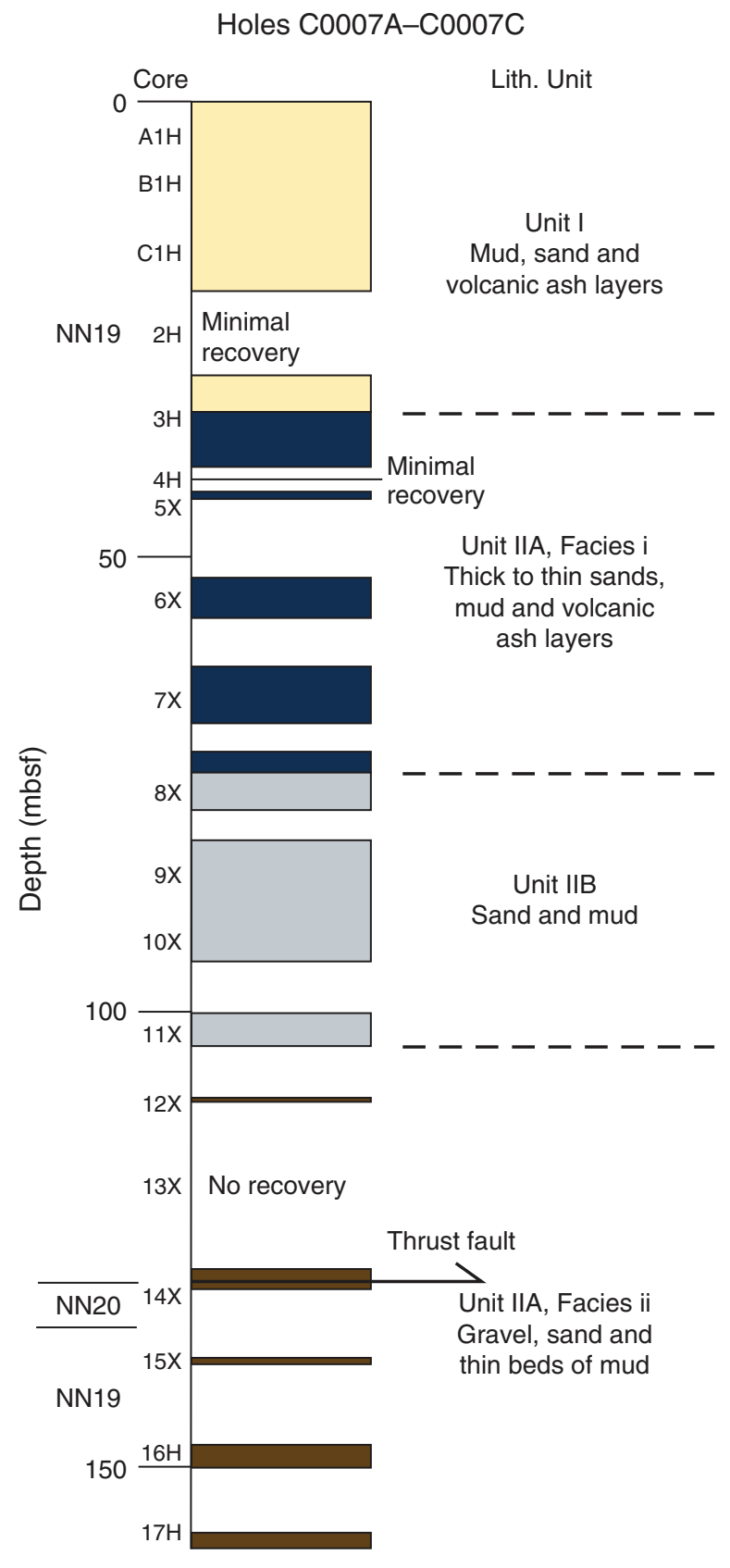


Figure F1 (continued). Core recovery for Hole C0007D.

Hole C0007D
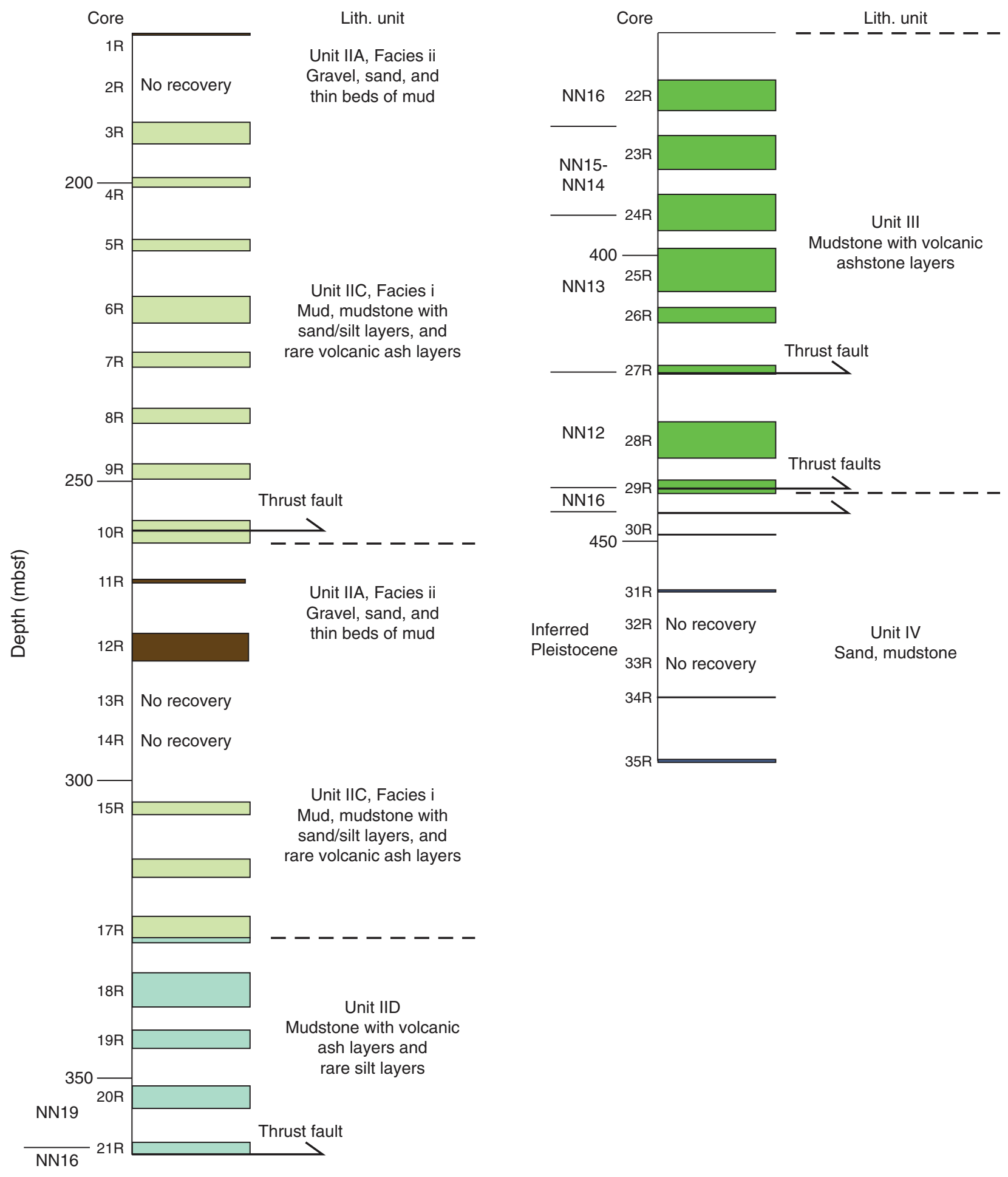

Unit IIC, Facies i Mud, mudstone with sand/silt layers, and are volcanic ash layers 
Figure F2. Sample locations in Cores 316-C0007C-17H and 316-C0007D-12R.

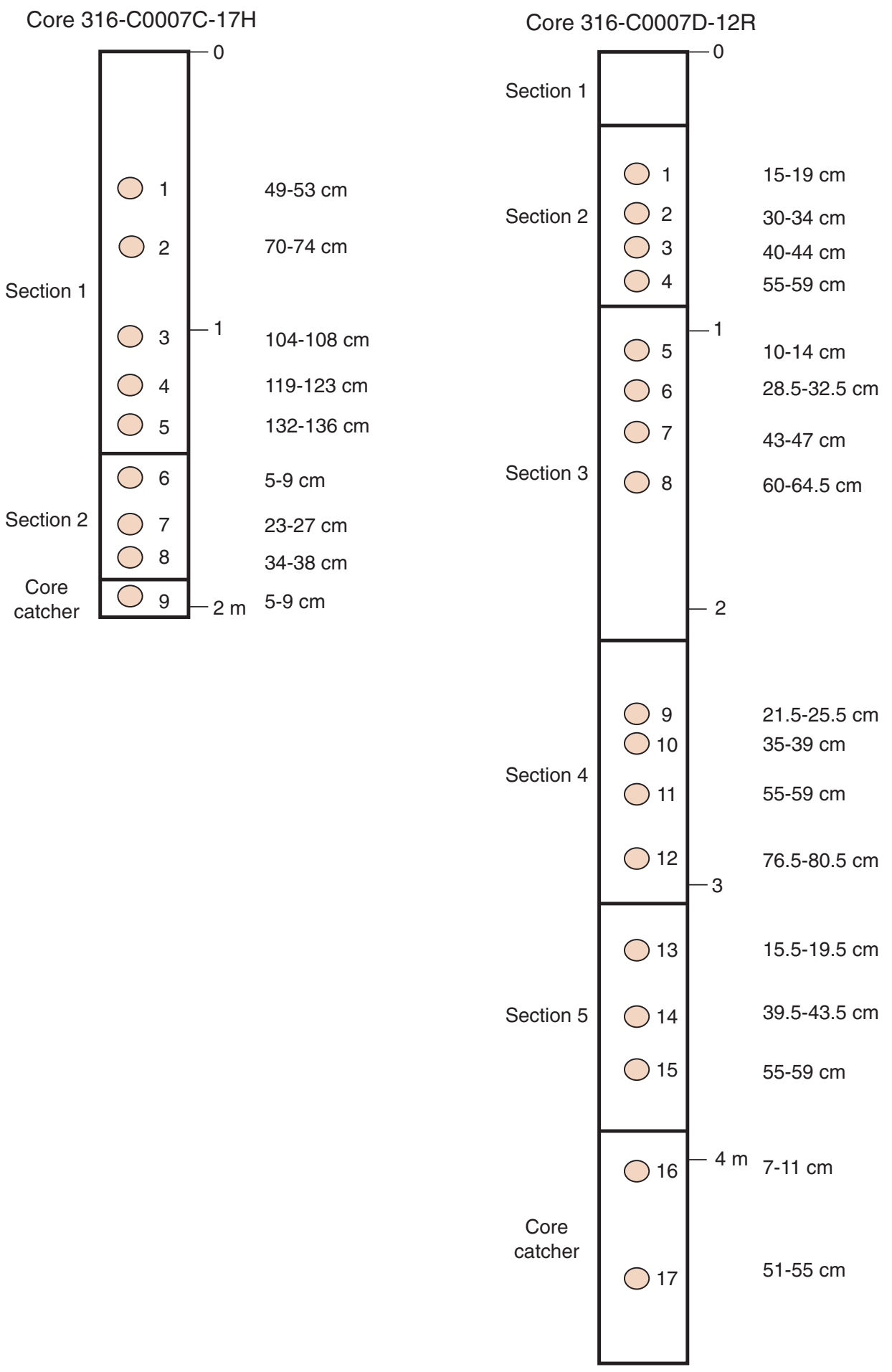


Figure F3. Clast counts for gravel samples from Site C0007. A. Core 316-C0007C-17H. (Continued on next two pages.)

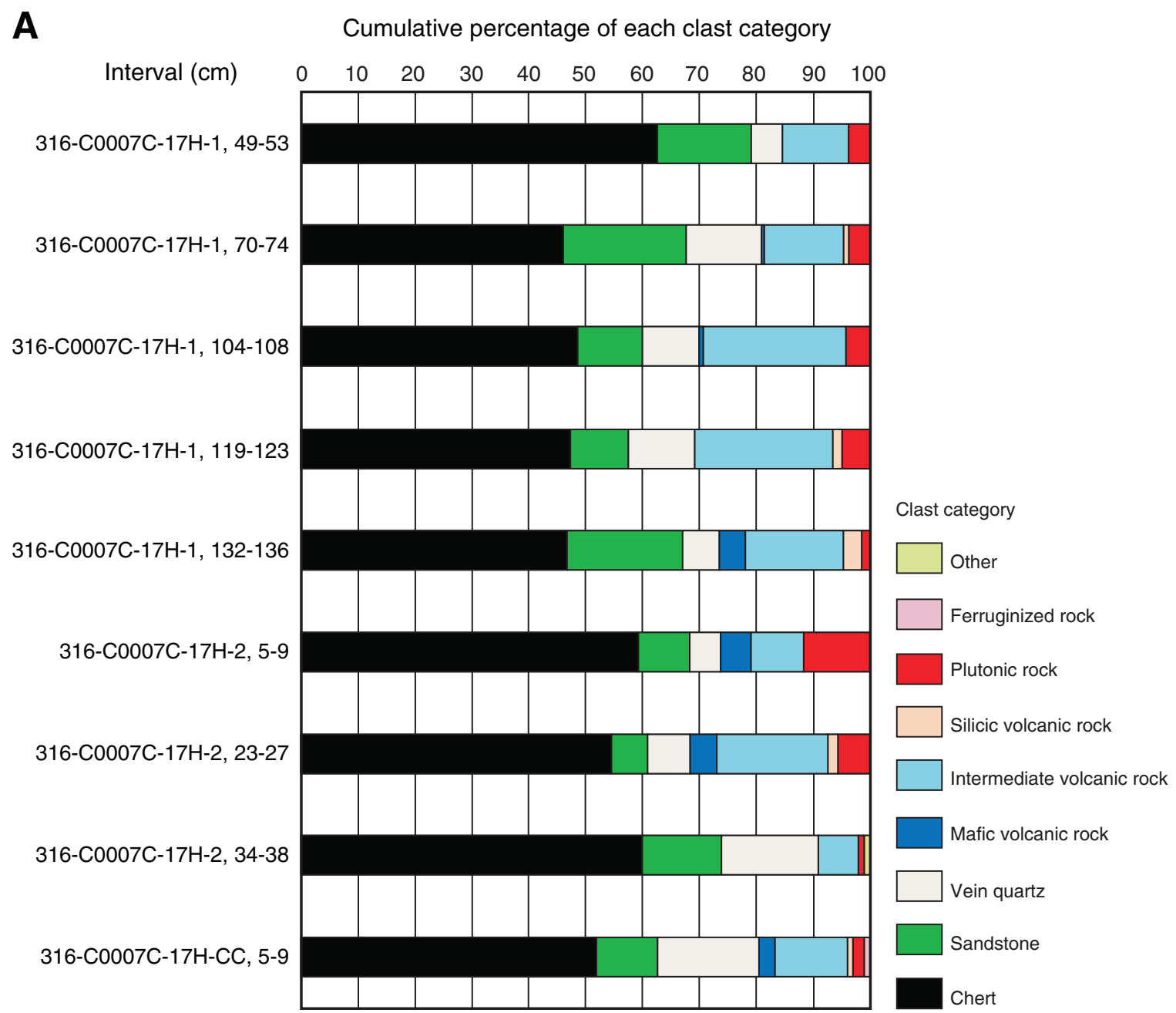


Figure F3 (continued). B. Core 316-C0007D-12R. (Continued on next page.)

B

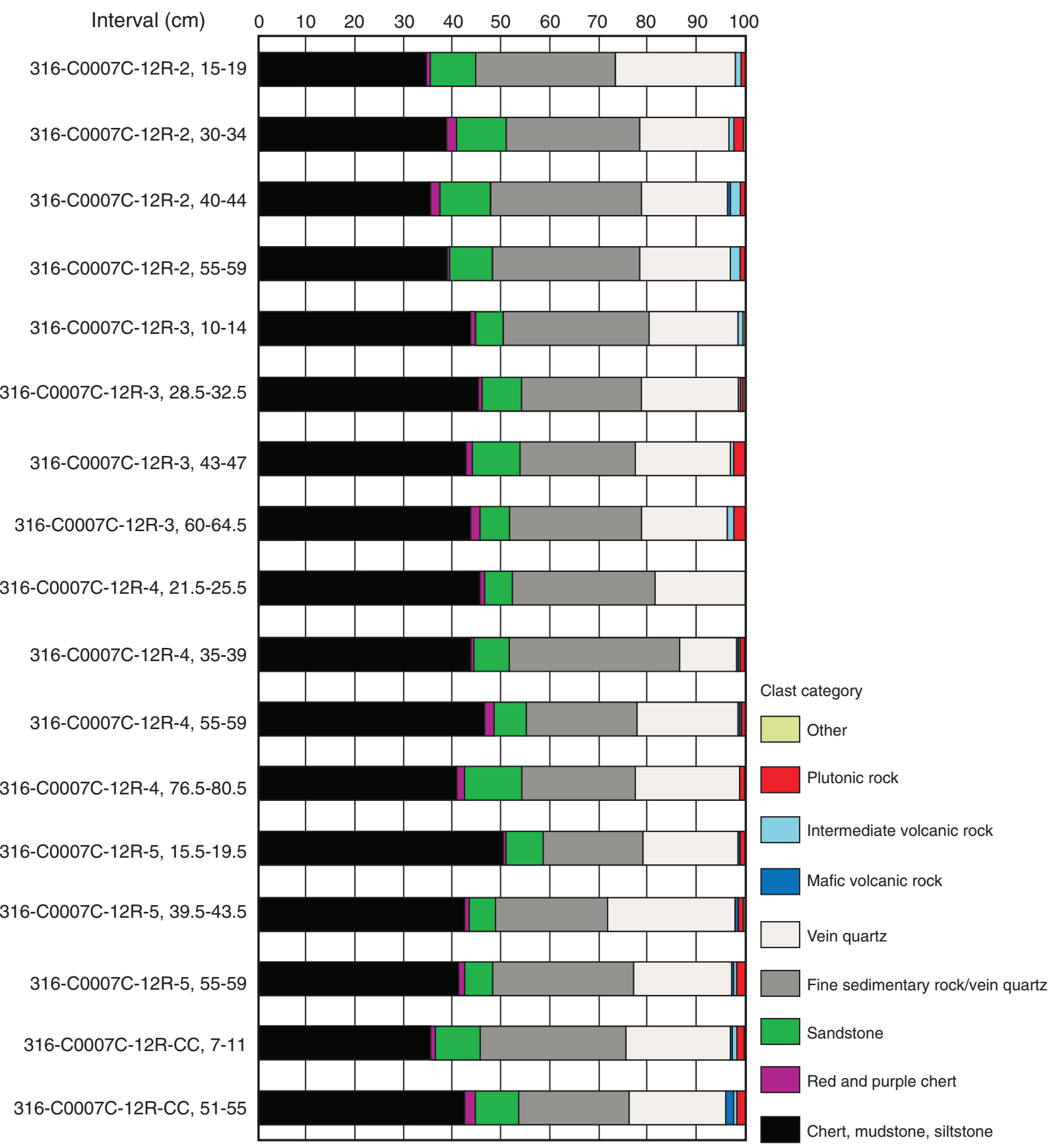


Figure F3 (continued). C. Core 316-C0007D-12R thin sections.

C

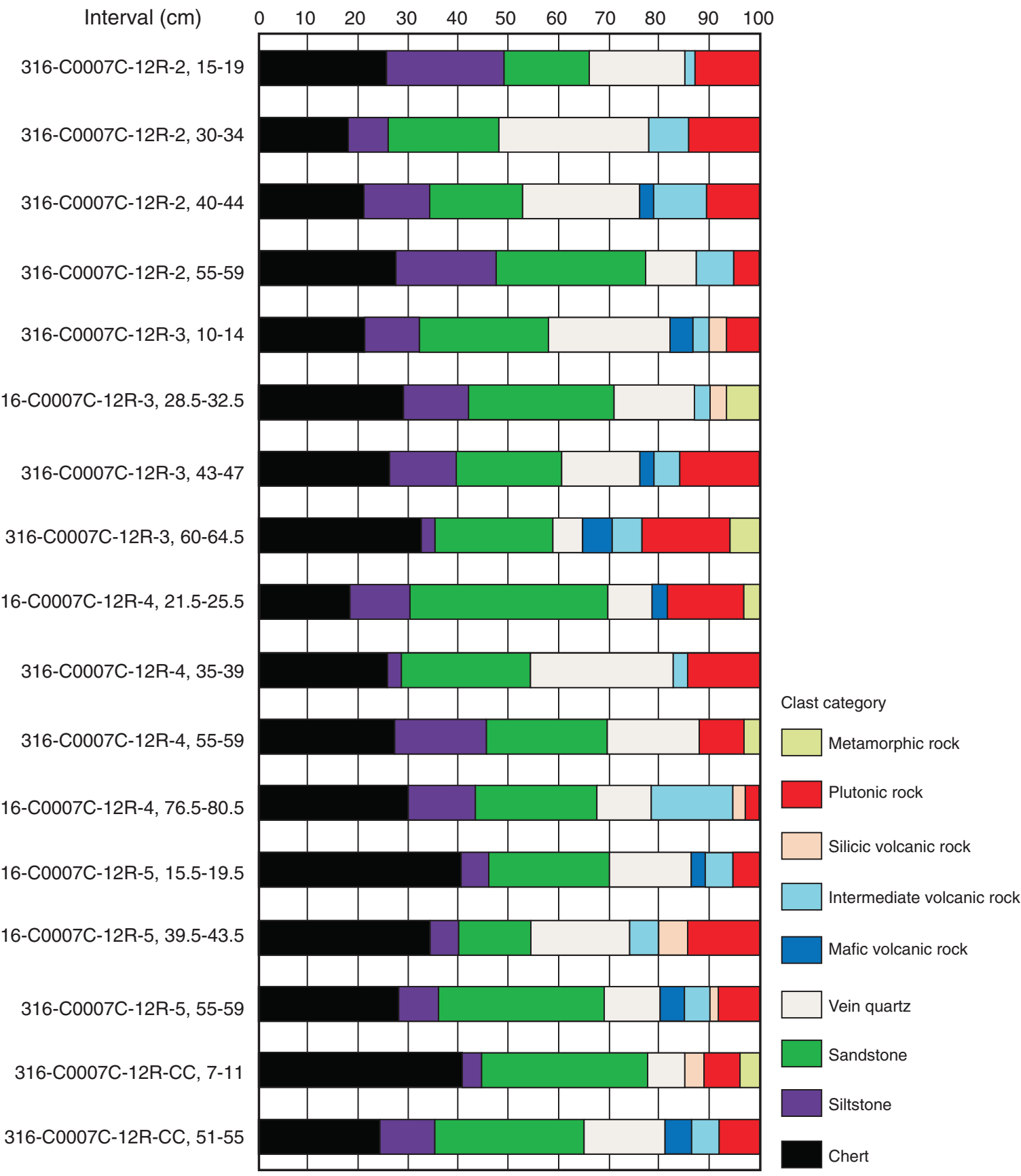


Figure F4. Photomicrographs of clast types in gravel (Sample 316-C0007D-12R-CC, 51-55 cm). A, B. Impure chert with quartz veins. C, D. Chert with quartz veins and poorly preserved radiolarians. E, F. Chert with radiolarian tests. G, H. Vein quartz. A, C, E, and G are under plane-polarized light and B, D, F, and H are under cross-polarized light.
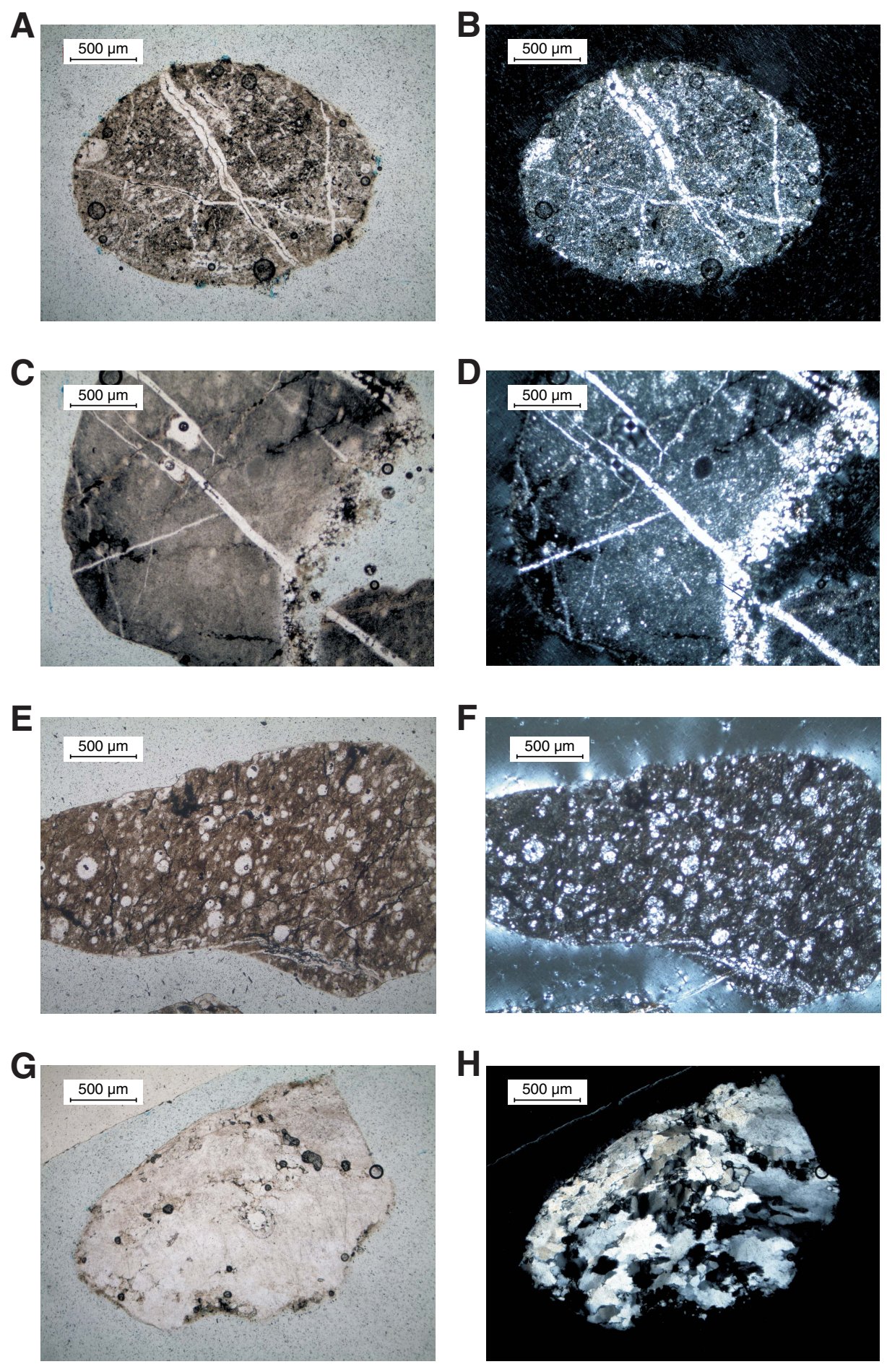
Figure F5. Photomicrographs of clast types in gravel. A, B. Very fine quartz sandstone. C, D. Fine to medium quartzose sandstone. E, F. Medium lithic sandstone with common intermediate volcanic rock fragments and quartz grains (Sample 316-C0007D-12R-4, 21-26 cm). G, H. Medium to coarse lithic sandstone dominated by intermediate volcanic rock fragments (A-D, G, and H: Sample 316-C0007D-12R-CC, 51-55 cm). A, C, E, and G are under plane-polarized light and B, D, F, and $\mathrm{H}$ are under cross-polarized light.
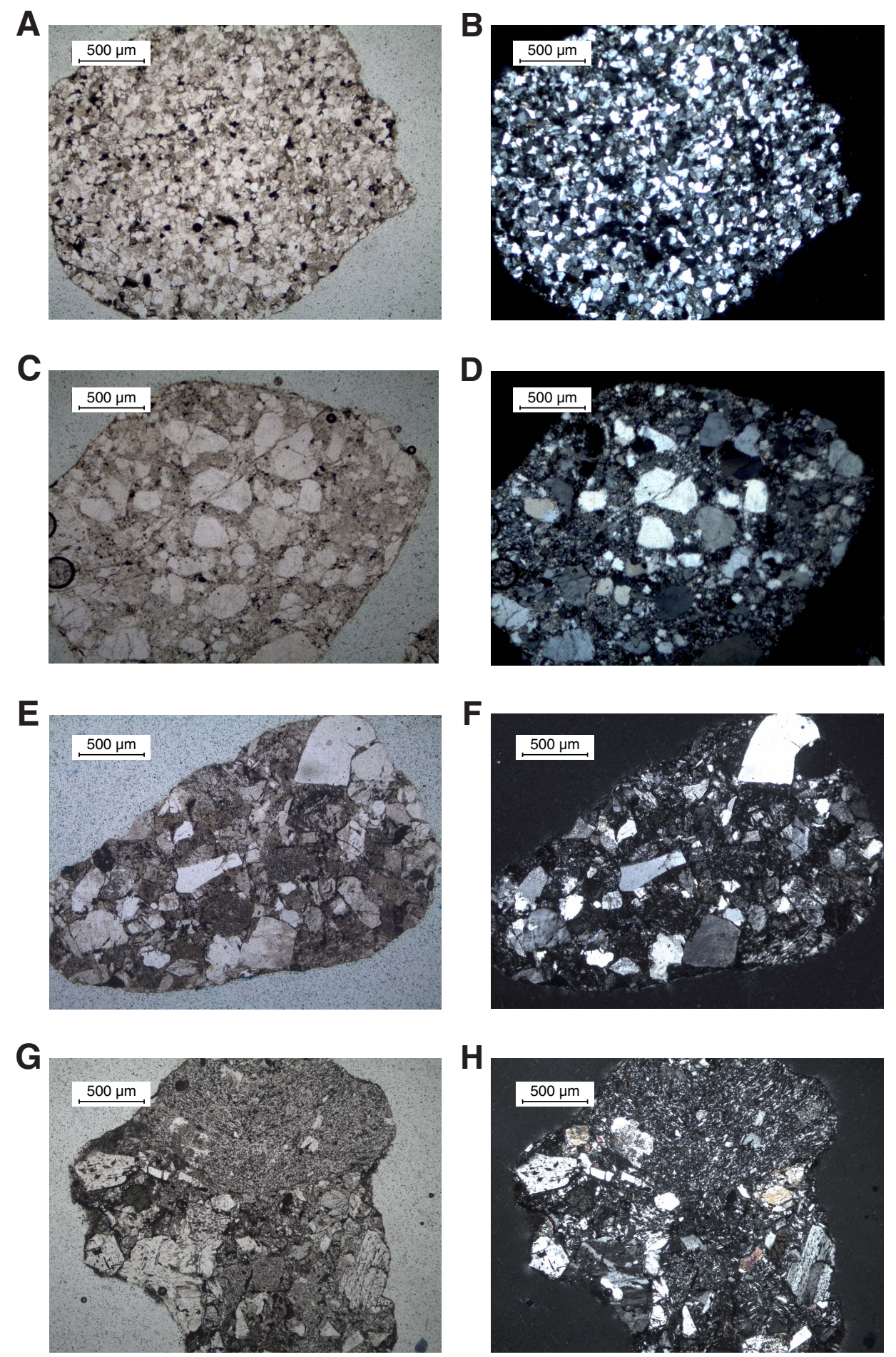
Figure F6. Photomicrographs of clast types in gravel. A, B. Porphyritic intermediate volcanic rock fragment (Sample 316-C0007D-12R-CC, 51-55 cm). C, D. Porphyritic intermediate volcanic rock fragment with plagioclase and orthopyroxene phenocrysts (Sample 316-C0007D-12R-2, 40-44 cm). E, F. Plutonic rock fragment with plagioclase, orthoclase, and quartz (Sample 316-C0007D-12R-4, 21-26 cm). G, H. Plutonic rock fragment with plagioclase, orthoclase, and quartz (Sample 316-C0007D-12R-4, 55-59 cm). A, C, E, and G are under planepolarized light and B, D, F, and $\mathrm{H}$ are under cross-polarized light.
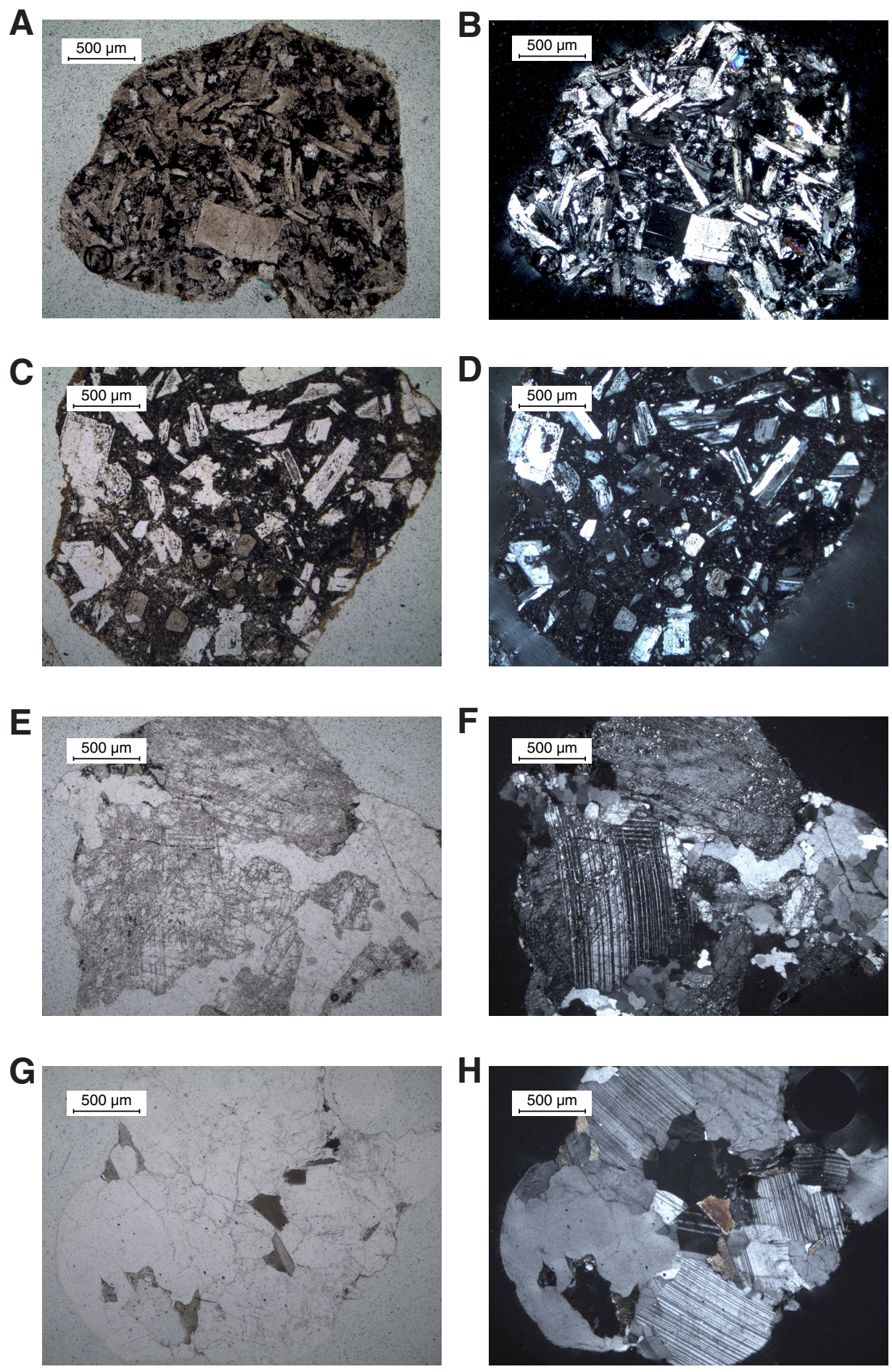
Figure F7. Photomicrographs of metasiltstone fragment. Note abundant biotite and a lack of foliation in (A) plane- and (B) cross-polarized light (Sample 316-C0007D-12R-4, 21-26 cm).
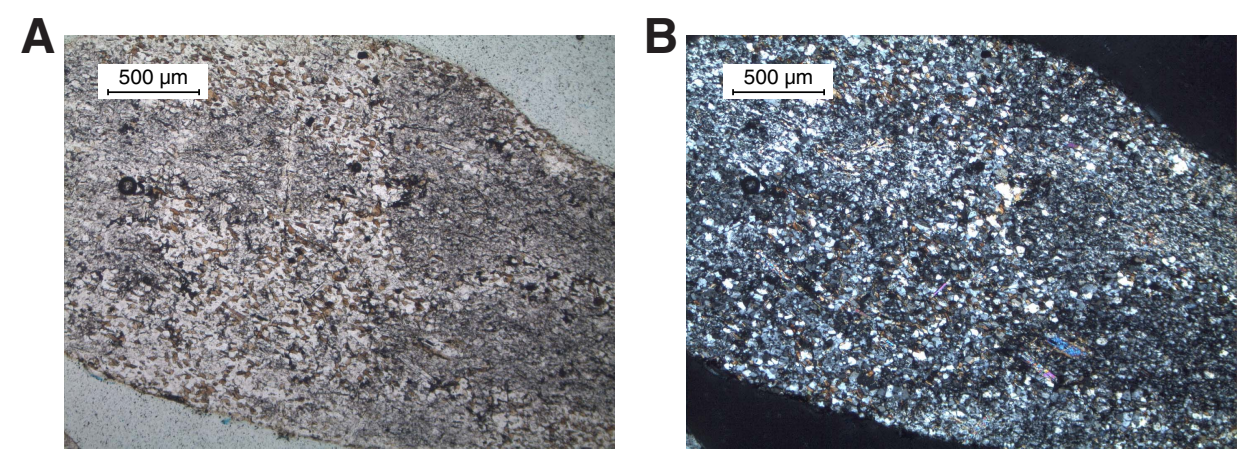
Table T1. Measurements of maximum grain diameters in gravel samples, Expedition 316.

\begin{tabular}{|c|c|c|c|c|c|c|c|c|c|}
\hline \multicolumn{10}{|c|}{ 316-C0007C- } \\
\hline $\begin{array}{l}\text { Core, section, } \\
\text { interval }(\mathrm{cm}) \text { : }\end{array}$ & $\begin{array}{l}17 \mathrm{H}-1 \\
49-53\end{array}$ & $\begin{array}{l}17 \mathrm{H}-1, \\
70-74\end{array}$ & $\begin{array}{c}\text { 17H-1, } \\
104-108\end{array}$ & $\begin{array}{c}17 \mathrm{H}-1 \\
119-123\end{array}$ & $\begin{array}{c}17 \mathrm{H}-1 \\
132-136\end{array}$ & $\begin{array}{c}17 \mathrm{H}-2 \\
5-9\end{array}$ & $\begin{array}{l}17 \mathrm{H}-2, \\
23-27\end{array}$ & $\begin{array}{l}17 \mathrm{H}-2 \\
34-38\end{array}$ & $\begin{array}{c}17 \mathrm{H}-\mathrm{CC}, \\
5-9\end{array}$ \\
\hline \multicolumn{10}{|l|}{ Diameter $(\mathrm{cm})$} \\
\hline 3.3 & 0 & 0 & 0 & 0 & 0 & 1 & 0 & 0 & 0 \\
\hline 2.2 & 0 & 0 & 0 & 0 & 0 & 1 & 1 & 1 & 0 \\
\hline 2.1 & 0 & 0 & 0 & 0 & 0 & 1 & 1 & 1 & 0 \\
\hline 2.0 & 0 & 0 & 0 & 0 & 1 & 0 & 1 & 0 & 1 \\
\hline 1.9 & 0 & 0 & 0 & 0 & 0 & 0 & 2 & 1 & 1 \\
\hline 1.8 & 0 & 0 & 0 & 0 & 1 & 0 & 0 & 1 & 1 \\
\hline 1.7 & 0 & 1 & 0 & 0 & 1 & 1 & 0 & 1 & 2 \\
\hline 1.6 & 0 & 0 & 0 & 1 & 3 & 2 & 0 & 2 & 0 \\
\hline 1.5 & 0 & 1 & 2 & 5 & 6 & 2 & 3 & 3 & 1 \\
\hline 1.4 & 0 & 0 & 1 & 5 & 5 & 0 & 0 & 0 & 3 \\
\hline 1.3 & 0 & 0 & 5 & 3 & 5 & 4 & 0 & 2 & 7 \\
\hline 1.2 & 0 & 2 & 14 & 8 & 3 & 4 & 3 & 4 & 7 \\
\hline 1.1 & 0 & 3 & 7 & 7 & 2 & 3 & 6 & 5 & 5 \\
\hline 1.0 & 3 & 6 & 15 & 18 & 12 & 7 & 16 & 8 & 8 \\
\hline 0.9 & 1 & 7 & 6 & 10 & 4 & 13 & 16 & 4 & 4 \\
\hline 0.8 & 2 & 10 & 17 & 15 & 3 & 13 & 19 & 13 & 12 \\
\hline 0.7 & 11 & 27 & 22 & 22 & 7 & 18 & 16 & 14 & 15 \\
\hline 0.6 & 11 & 18 & 12 & 12 & 5 & 9 & 7 & 16 & 9 \\
\hline 0.5 & 40 & 32 & 16 & 18 & 5 & 15 & 12 & 11 & 15 \\
\hline 0.4 & 51 & 49 & 12 & 6 & & 8 & 1 & 9 & 2 \\
\hline 0.3 & 85 & 44 & 11 & 9 & 1 & 9 & 4 & 4 & 9 \\
\hline Total: & 204 & 200 & 140 & 139 & 64 & 111 & 108 & 100 & 102 \\
\hline
\end{tabular}


Table T2. Average maximum grain diameters in gravel samples, Expedition 316.

\begin{tabular}{lcc}
\hline $\begin{array}{c}\text { Core, section, } \\
\text { interval }(\mathrm{cm})\end{array}$ & $\begin{array}{c}\text { Number of } \\
\text { clasts }\end{array}$ & $\begin{array}{c}\text { Average diameter } \\
(\mathrm{cm})\end{array}$ \\
\hline $316-\mathrm{C} 0007 \mathrm{C}-$ & & \\
$17 \mathrm{H}-1,49-3$ & 204 & 0.42 \\
$17 \mathrm{H}-1,70-4$ & 200 & 0.54 \\
$17 \mathrm{H}-1,104-08$ & 140 & 0.77 \\
$17 \mathrm{H}-1,119-23$ & 139 & 0.81 \\
$17 \mathrm{H}-1,132-36$ & 64 & 1.05 \\
$17 \mathrm{H}-2,5-9$ & 111 & 0.80 \\
$17 \mathrm{H}-2,23-27$ & 108 & 0.86 \\
$17 \mathrm{H}-2,34-38$ & 100 & 0.82 \\
$17 \mathrm{H}-\mathrm{CC}, 5-9$ & 102 & 0.84 \\
\hline
\end{tabular}


Table T3. Raw clast count and percentage clast count for gravel samples, Expedition 316.

\begin{tabular}{|c|c|c|c|c|c|c|c|c|c|c|c|c|c|}
\hline $\begin{array}{l}\text { Core, section, } \\
\text { interval }(\mathrm{cm})\end{array}$ & $\begin{array}{l}\text { Chert (black, } \\
\text { gray, light } \\
\text { yellow-brown, } \\
\text { clear) }\end{array}$ & Sandstone & $\begin{array}{l}\text { Vein } \\
\text { quartz }\end{array}$ & $\begin{array}{l}\text { Mafic } \\
\text { volcanic } \\
\text { rock }\end{array}$ & $\begin{array}{l}\text { Intermediate } \\
\text { volcanic rock }\end{array}$ & $\begin{array}{l}\text { Silicic } \\
\text { volcanic } \\
\text { rock }\end{array}$ & $\begin{array}{l}\text { Plutonic } \\
\text { rock }\end{array}$ & $\begin{array}{l}\text { Ferruginized } \\
\text { rock }\end{array}$ & Other & Total & Shell & $\begin{array}{l}\text { Gray-brown soft } \\
\text { mudstone } \\
\text { (intraformational) }\end{array}$ & Total \\
\hline \multicolumn{14}{|l|}{ 316-C0007C- } \\
\hline $17 \mathrm{H}-1,49-53$ & 449 & 121 & 38 & 1 & 83 & 0 & 27 & 0 & 0 & 719 & 2 & 71 & 792 \\
\hline $17 \mathrm{H}-1,70-74$ & 187 & 88 & 53 & 2 & 57 & 4 & 15 & 0 & 0 & 406 & 1 & 55 & 462 \\
\hline 17H-1, 104-108 & 68 & 16 & 14 & 1 & 35 & 0 & 6 & 0 & 0 & 140 & 0 & 0 & 140 \\
\hline 17H-1, 119-123 & 66 & 14 & 16 & 0 & 34 & 2 & 7 & 0 & 0 & 139 & 0 & 6 & 145 \\
\hline $17 \mathrm{H}-1,132-136$ & 30 & 13 & 4 & 3 & 11 & 2 & 1 & 0 & 0 & 64 & 0 & 1 & 65 \\
\hline $17 \mathrm{H}-2,5-9$ & 66 & 10 & 6 & 6 & 10 & 0 & 13 & 0 & 0 & 111 & 0 & 1 & 112 \\
\hline $17 \mathrm{H}-2,23-27$ & 59 & 7 & 8 & 5 & 21 & 2 & 6 & 0 & 0 & 108 & 0 & 0 & 108 \\
\hline $17 \mathrm{H}-2,34-38$ & 60 & 14 & 17 & 0 & 7 & 0 & 1 & 0 & 1 & 100 & 1 & 0 & 101 \\
\hline $17 \mathrm{H}-\mathrm{CC}, 5-9$ & 53 & 11 & 18 & 3 & 13 & 1 & 2 & 1 & 0 & 102 & 0 & 2 & 104 \\
\hline \multicolumn{14}{|l|}{ Percentage: } \\
\hline $17 \mathrm{H}-1,49-53$ & 62 & 17 & 5 & 0 & 12 & 0 & 4 & 0 & 0 & 100 & & & \\
\hline $17 \mathrm{H}-1,70-74$ & 46 & 22 & 13 & 0 & 14 & 1 & 4 & 0 & 0 & 100 & & & \\
\hline $17 \mathrm{H}-1,104-108$ & 49 & 11 & 10 & 1 & 25 & 0 & 4 & 0 & 0 & 100 & & & \\
\hline $17 \mathrm{H}-1,119-123$ & 47 & 10 & 12 & 0 & 24 & 1 & 5 & 0 & 0 & 100 & & & \\
\hline $17 \mathrm{H}-1,132-136$ & 47 & 20 & 6 & 5 & 17 & 3 & 2 & 0 & 0 & 100 & & & \\
\hline $17 \mathrm{H}-2,5-9$ & 59 & 9 & 5 & 5 & 9 & 0 & 12 & 0 & 0 & 100 & & & \\
\hline $17 \mathrm{H}-2,23-27$ & 55 & 6 & 7 & 5 & 19 & 2 & 6 & 0 & 0 & 100 & & & \\
\hline $17 \mathrm{H}-2,34-38$ & 60 & 14 & 17 & 0 & 7 & 0 & 1 & 0 & 1 & 100 & & & \\
\hline $17 \mathrm{H}-\mathrm{CC}, 5-9$ & 52 & 11 & 18 & 3 & 13 & 1 & 2 & 1 & 0 & 100 & & & \\
\hline
\end{tabular}


Table T4. Raw clast count and percentage clast count for gravel samples, Expedition 316.

\begin{tabular}{|c|c|c|c|c|c|c|c|c|c|c|c|c|c|}
\hline $\begin{array}{l}\text { Core, section, } \\
\text { interval }(\mathrm{cm})\end{array}$ & $\begin{array}{l}\text { Chert, } \\
\text { mudstone, } \\
\text { siltstone }\end{array}$ & $\begin{array}{c}\text { Red and } \\
\text { purple } \\
\text { chert }\end{array}$ & Sandstone & $\begin{array}{c}\text { Fine } \\
\text { sedimentary } \\
\text { rock/vein } \\
\text { quartz }\end{array}$ & $\begin{array}{l}\text { Vein } \\
\text { quartz }\end{array}$ & $\begin{array}{l}\text { Mafic } \\
\text { volcanic } \\
\text { rock }\end{array}$ & $\begin{array}{l}\text { Intermediate } \\
\text { volcanic rock }\end{array}$ & $\begin{array}{l}\text { Plutonic } \\
\text { rock }\end{array}$ & Other & Total & Shell & $\begin{array}{l}\text { Gray-brown soft } \\
\text { mudstone } \\
\text { (intraformational) }\end{array}$ & Total \\
\hline \multicolumn{14}{|l|}{$\begin{array}{l}\text { 316-C0007D- } \\
\text { Raw count: }\end{array}$} \\
\hline $12 \mathrm{R}-2,15-19$ & 148 & 4 & 40 & 122 & 105 & 1 & 5 & 3 & 0 & 428 & 1 & 4 & 433 \\
\hline $12 \mathrm{R}-2,2,30-34$ & 162 & 10 & 42 & 115 & 76 & 0 & 5 & 8 & 1 & 419 & & 13 & 432 \\
\hline $12 \mathrm{R}-2,2,40-44$ & 157 & 9 & 46 & 136 & 78 & 3 & 9 & 4 & 0 & 442 & & 15 & 457 \\
\hline $12 \mathrm{R}-2,2,55-59$ & 181 & 2 & 41 & 140 & 86 & 0 & 10 & 4 & 0 & 464 & & 2 & 466 \\
\hline $12 \mathrm{R}-2,3,10-14$ & 186 & 4 & 24 & 128 & 77 & 0 & 4 & 0 & 2 & 425 & & 13 & 438 \\
\hline $12 \mathrm{R}-2,3,28.5-32.5$ & 187 & 3 & 34 & 101 & 83 & 0 & 1 & 3 & 1 & 413 & & 4 & 417 \\
\hline $12 \mathrm{R}-2,3,43-47$ & 199 & 5 & 46 & 110 & 90 & 0 & 4 & 9 & 1 & 464 & & 7 & 471 \\
\hline $12 \mathrm{R}-2,3,60-64.5$ & 207 & 8 & 29 & 127 & 83 & 0 & 7 & 10 & 0 & 471 & & 2 & 473 \\
\hline $12 \mathrm{R}-2,4,21.5-25.5$ & 202 & 4 & 26 & 129 & 82 & 0 & 0 & 0 & 0 & 443 & & 1 & 444 \\
\hline $12 \mathrm{R}-2,4,35-39$ & 221 & 3 & 37 & 176 & 60 & 2 & 1 & 5 & 0 & 505 & & 9 & 514 \\
\hline $12 \mathrm{R}-2,4,55-59$ & 275 & 11 & 40 & 135 & 122 & 2 & 1 & 4 & 1 & 591 & & 6 & 597 \\
\hline $12 \mathrm{R}-2,4,76.5-80.5$ & 191 & 7 & 55 & 109 & 101 & 0 & 0 & 4 & 0 & 467 & & 12 & 479 \\
\hline $12 \mathrm{R}-2,5,15.5-19.5$ & 334 & 4 & 50 & 137 & 129 & 3 & 0 & 6 & 0 & 663 & 1 & 3 & 667 \\
\hline $12 \mathrm{R}-2,5,39.5-43.5$ & 233 & 5 & 29 & 125 & 143 & 3 & 0 & 6 & 2 & 546 & & 5 & 551 \\
\hline $12 \mathrm{R}-2,5,55-59$ & 269 & 8 & 37 & 189 & 132 & 2 & 5 & 9 & 1 & 652 & & 5 & 657 \\
\hline $12 \mathrm{R}-2, \mathrm{CC}, 7-11$ & 180 & 5 & 45 & 152 & 109 & 1 & 4 & 9 & 0 & 505 & & 7 & 512 \\
\hline $12 \mathrm{R}-2, \mathrm{CC}, 51-55$ & 193 & 10 & 40 & 104 & 89 & 7 & 4 & 7 & 0 & 454 & & 5 & 459 \\
\hline \multicolumn{14}{|l|}{ Percentage: } \\
\hline $12 \mathrm{R}-2,2,15-19$ & 35 & 1 & 9 & 29 & 25 & 0 & 1 & 1 & 0 & 100 & & & \\
\hline $12 \mathrm{R}-2,2,30-34$ & 39 & 2 & 10 & 27 & 18 & 0 & 1 & 2 & 0 & 100 & & & \\
\hline $12 \mathrm{R}-2,2,40-44$ & 36 & 2 & 10 & 31 & 18 & 1 & 2 & 1 & 0 & 100 & & & \\
\hline $12 \mathrm{R}-2,2,55-59$ & 39 & 0 & 9 & 30 & 19 & 0 & 2 & 1 & 0 & 100 & & & \\
\hline $12 \mathrm{R}-2,3,10-14$ & 44 & 1 & 6 & 30 & 18 & 0 & 1 & 0 & 0 & 100 & & & \\
\hline $12 \mathrm{R}-2,3,28.5-32.5$ & 45 & 1 & 8 & 24 & 20 & 0 & 0 & 1 & 0 & 100 & & & \\
\hline $12 \mathrm{R}-2,3,43-47$ & 43 & 1 & 10 & 24 & 19 & 0 & 1 & 2 & 0 & 100 & & & \\
\hline $12 \mathrm{R}-2,3,60-64.5$ & 44 & 2 & 6 & 27 & 18 & 0 & 1 & 2 & 0 & 100 & & & \\
\hline $12 \mathrm{R}-2,4,21.5-25.5$ & 46 & 1 & 6 & 29 & 19 & 0 & 0 & 0 & 0 & 100 & & & \\
\hline $12 \mathrm{R}-2,4,35-39$ & 44 & 1 & 7 & 35 & 12 & 0 & 0 & 1 & 0 & 100 & & & \\
\hline $12 \mathrm{R}-2,4,55-59$ & 47 & 2 & 7 & 23 & 21 & 0 & 0 & 1 & 0 & 100 & & & \\
\hline $12 \mathrm{R}-2,4,76.5-80.5$ & 41 & 1 & 12 & 23 & 22 & 0 & 0 & 1 & 0 & 100 & & & \\
\hline $12 \mathrm{R}-2,5,15.5-19.5$ & 50 & 1 & 8 & 21 & 19 & 0 & 0 & 1 & 0 & 100 & & & \\
\hline $12 \mathrm{R}-2,5,39.5-43.5$ & 43 & 1 & 5 & 23 & 26 & 1 & 0 & 1 & 0 & 100 & & & \\
\hline $12 \mathrm{R}-2,5,55-59$ & 41 & 1 & 6 & 29 & 20 & 0 & 1 & 1 & 0 & 100 & & & \\
\hline $12 \mathrm{R}-2, \mathrm{CC}, 7-11$ & 36 & 1 & 9 & 30 & 22 & 0 & 1 & 2 & 0 & 100 & & & \\
\hline $12 \mathrm{R}-2, \mathrm{CC}, 51-55$ & 43 & 2 & 9 & 23 & 20 & 2 & 1 & 2 & 0 & 100 & & & \\
\hline
\end{tabular}


Table T5. Raw clast count and percentage clast count for thin sections of gravel samples, Expedition 316. (See table note.)

\begin{tabular}{|c|c|c|c|c|c|c|c|c|c|c|c|c|c|c|c|c|}
\hline $\begin{array}{l}\text { Core, section, } \\
\text { interval }(\mathrm{cm})\end{array}$ & Chert & Siltstone & Sandstone & $\begin{array}{l}\text { Vein } \\
\text { quartz }\end{array}$ & $\begin{array}{c}\text { Mafic } \\
\text { volcanic } \\
\text { rock }\end{array}$ & $\begin{array}{l}\text { Intermediate } \\
\text { volcanic rock }\end{array}$ & $\begin{array}{l}\text { Silicic } \\
\text { volcanic } \\
\text { rock }\end{array}$ & $\begin{array}{l}\text { Plutonic } \\
\text { rock }\end{array}$ & $\begin{array}{l}\text { Metamorphic } \\
\text { rock }\end{array}$ & Total & $\begin{array}{l}\text { Gray-brown soft } \\
\text { mudstone } \\
\text { (intraformational) }\end{array}$ & & & & & \\
\hline \multicolumn{17}{|l|}{$\begin{array}{l}\text { 316-C0007D- } \\
\text { Raw count: }\end{array}$} \\
\hline $12 \mathrm{R}-2,15-19$ & 12 & 11 & 8 & 9 & 0 & 1 & 0 & 6 & 0 & 47 & 3 & & & & & \\
\hline $12 \mathrm{R}-2,30-34$ & 9 & 4 & 11 & 15 & 0 & 4 & 0 & 7 & 0 & 50 & 2 & & & & & \\
\hline $12 \mathrm{R}-2,40-44$ & 8 & 5 & 7 & 9 & 1 & 4 & 0 & 4 & 0 & 38 & 9 & & & & & \\
\hline $12 \mathrm{R}-2,55-59$ & 11 & 8 & 12 & 4 & 0 & 3 & 0 & 2 & 0 & 40 & 4 & & & & & \\
\hline $12 \mathrm{R}-3,10-14$ & 19 & 10 & 23 & 22 & 4 & 3 & 3 & 6 & 0 & 90 & 4 & & & & & \\
\hline $12 \mathrm{R}-3,28.5-32.5$ & 9 & 4 & 9 & 5 & 0 & 1 & 1 & 0 & 2 & 31 & 0 & & & & & \\
\hline $12 \mathrm{R}-3,43-47$ & 10 & 5 & 8 & 6 & 1 & 2 & 0 & 6 & 0 & 38 & 1 & & & & & \\
\hline $12 \mathrm{R}-3,60-64.5$ & 11 & 1 & 8 & 2 & 2 & 2 & 0 & 6 & 2 & 34 & 1 & & & & & \\
\hline $12 \mathrm{R}-4,21.5-25.5$ & 6 & 4 & 13 & 3 & 1 & 0 & 0 & 5 & 1 & 33 & 2 & & & & & \\
\hline $12 \mathrm{R}-4,35-39$ & 9 & 1 & 9 & 10 & 0 & 1 & 0 & 5 & 0 & 35 & 1 & & & & & \\
\hline $12 \mathrm{R}-4,55-59$ & 9 & 6 & 8 & 6 & 0 & 0 & 0 & 3 & 1 & 33 & 2 & & & & & \\
\hline $12 \mathrm{R}-4,76.5-80.5$ & 11 & 5 & 9 & 4 & 0 & 6 & 1 & 1 & 0 & 37 & 1 & & & & & \\
\hline $12 \mathrm{R}-5,15.5-19.5$ & 15 & 2 & 9 & 6 & 1 & 2 & 0 & 2 & 0 & 37 & 2 & & & & & \\
\hline $12 \mathrm{R}-5,39.5-43.5$ & 12 & 2 & 5 & 7 & 0 & 2 & 2 & 5 & 0 & 35 & 0 & & & & & \\
\hline $12 \mathrm{R}-5,55-59$ & 17 & 5 & 20 & 7 & 3 & 3 & 1 & 5 & 0 & 61 & 3 & & & & & \\
\hline 12R-CC, 7-11 & 11 & 1 & 9 & 2 & 0 & 0 & 1 & 2 & 1 & 27 & 0 & & & & & \\
\hline $12 \mathrm{R}-\mathrm{CC}, 51-55$ & 9 & 4 & 11 & 6 & 2 & 2 & 0 & 3 & 0 & 37 & 1 & & & & & \\
\hline Percentage: & & & & & & & & & & & & SRF & VQ & PRF & VRF & MRF \\
\hline $12 \mathrm{R}-2,15-19$ & 26 & 23 & 17 & 19 & 0 & 2 & 0 & 13 & 0 & 100 & & 66 & 19 & 13 & 2 & 0 \\
\hline $12 \mathrm{R}-2,30-34$ & 18 & 8 & 22 & 30 & 0 & 8 & 0 & 14 & 0 & 100 & & 48 & 30 & 14 & 8 & 0 \\
\hline $12 \mathrm{R}-2,40-44$ & 21 & 13 & 18 & 24 & 3 & 11 & 0 & 11 & 0 & 100 & & 53 & 24 & 11 & 13 & 0 \\
\hline $12 \mathrm{R}-2,55-59$ & 28 & 20 & 30 & 10 & 0 & 8 & 0 & 5 & 0 & 100 & & 78 & 10 & 5 & 8 & 0 \\
\hline $12 \mathrm{R}-3,10-14$ & 21 & 11 & 26 & 24 & 4 & 3 & 3 & 7 & 0 & 100 & & 58 & 24 & 7 & 11 & 0 \\
\hline $12 \mathrm{R}-3,28.5-32.5$ & 29 & 13 & 29 & 16 & 0 & 3 & 3 & 0 & 6 & 100 & & 71 & 16 & 0 & 6 & 6 \\
\hline $12 \mathrm{R}-3,43-47$ & 26 & 13 & 21 & 16 & 3 & 5 & 0 & 16 & 0 & 100 & & 61 & 16 & 16 & 8 & 0 \\
\hline $12 R-3,60-64.5$ & 32 & 3 & 24 & 6 & 6 & 6 & 0 & 18 & 6 & 100 & & 59 & 6 & 18 & 12 & 6 \\
\hline $12 \mathrm{R}-4,21.5-25.5$ & 18 & 12 & 39 & 9 & 3 & 0 & 0 & 15 & 3 & 100 & & 70 & 9 & 15 & 3 & 3 \\
\hline $12 \mathrm{R}-4,35-39$ & 26 & 3 & 26 & 29 & 0 & 3 & 0 & 14 & 0 & 100 & & 54 & 29 & 14 & 3 & 0 \\
\hline $12 \mathrm{R}-4,55-59$ & 27 & 18 & 24 & 18 & 0 & 0 & 0 & 9 & 3 & 100 & & 70 & 18 & 9 & 0 & 3 \\
\hline $12 \mathrm{R}-4,76.5-80.5$ & 30 & 14 & 24 & 11 & 0 & 16 & 3 & 3 & 0 & 100 & & 68 & 11 & 3 & 19 & 0 \\
\hline $12 \mathrm{R}-5,15.5-19.5$ & 41 & 5 & 24 & 16 & 3 & 5 & 0 & 5 & 0 & 100 & & 70 & 16 & 5 & 8 & 0 \\
\hline $12 \mathrm{R}-5,39.5-43.5$ & 34 & 6 & 14 & 20 & 0 & 6 & 6 & 14 & 0 & 100 & & 54 & 20 & 14 & 11 & 0 \\
\hline $12 \mathrm{R}-5,55-59$ & 28 & 8 & 33 & 11 & 5 & 5 & 2 & 8 & 0 & 100 & & 69 & 11 & 8 & 11 & 0 \\
\hline 12R-CC, 7-11 & 41 & 4 & 33 & 7 & 0 & 0 & 4 & 7 & 4 & 100 & & 78 & 7 & 7 & 4 & 4 \\
\hline $12 \mathrm{RCC}, 51-55$ & 24 & 11 & 30 & 16 & 5 & 5 & 0 & 8 & 0 & 100 & & 65 & 16 & 8 & 11 & 0 \\
\hline
\end{tabular}

Note: $\mathrm{SRF}=$ sedimentary rock fragment, $\mathrm{VQ}=$ vein quartz, $\mathrm{PRF}=$ plutonic rock fragment, $\mathrm{VRF}=$ volcanic rock fragment, $\mathrm{MRF}=$ metamorphic rock fragment. 
Table T6. X-ray fluorescence major chemical analyses of six representative clasts, Expedition 316, Sample C0007C-17H, CC, 5-9 cm.

\begin{tabular}{lrrrrrr}
\hline $\begin{array}{l}\text { Major element } \\
\text { oxide (wt\%) }\end{array}$ & Chert & Vein quartz & Siltstone & $\begin{array}{r}\text { Volcanic } \\
\text { rock }\end{array}$ & $\begin{array}{r}\text { Metamorphic } \\
\text { rock }\end{array}$ & Sandstone \\
\hline $\mathrm{SiO}_{2}$ & 96.11 & 94.31 & 82.76 & 72.15 & 70.73 & 76.55 \\
$\mathrm{TiO}_{2}$ & 0.03 & 0.04 & 0.15 & 0.40 & 0.33 & 0.33 \\
$\mathrm{Al}_{2} \mathrm{O}_{3}$ & 0.89 & 1.99 & 7.52 & 12.56 & 12.94 & 10.67 \\
$\mathrm{Fe}_{2} \mathrm{O}_{3}$ & 0.20 & 0.18 & 0.92 & 2.84 & 3.09 & 3.45 \\
$\mathrm{MnO}$ & 0.00 & 0.01 & 0.02 & 0.03 & 0.04 & 0.02 \\
$\mathrm{MgO}$ & 0.01 & 0.05 & 0.23 & 0.85 & 0.97 & 1.03 \\
$\mathrm{Na} O$ & 0.16 & 0.17 & 2.52 & 3.64 & 3.43 & 2.49 \\
$\mathrm{CaO}$ & 0.06 & 0.06 & 0.24 & 0.59 & 0.73 & 0.26 \\
$\mathrm{~K}_{2} \mathrm{O}$ & 0.24 & 0.39 & 1.50 & 2.55 & 2.88 & 2.23 \\
$\mathrm{P}_{2} \mathrm{O}_{5}$ & 0.03 & 0.02 & 0.07 & 0.13 & 0.04 & 0.07 \\
$\mathrm{SO}_{3}$ & 0.06 & 0.01 & 0.34 & 0.42 & 1.77 & 0.87 \\
\hline \multicolumn{1}{r}{ Total: } & 97.79 & 97.23 & 96.28 & 96.15 & 96.95 & 97.98 \\
\hline
\end{tabular}

